\title{
MÉTODO INOVADOR DE ENSINO PARA A PRÁTICA DE SUTURAS DE PELE
}

\section{INNOVATIVE TEACHING MODEL FOR THE PRACTICE OF SKIN SUTURING}

\author{
Emannuella Araújo de Oliveira ${ }^{1}$ \\ Lenilda Austrilino ${ }^{2}$
}

\begin{abstract}
Resumo: Este trabalho tem como objetivo mostrar potencialidades de um método inovador de ensino, para prática de sutura de pele, usando materiais orgânicos e sintéticos. Trata-se de um estudo de caso com discentes de medicina, participantes de oficina realizada aplicando técnicas básicas de sutura de pele, com treinamento simulado em três consistências de tecidos. Os dados coletados com questionários e observações avaliando desempenho discente e efetividade do método. As suturas realizadas em EVA, berinjela e língua de boi utilizando ponto simples, simples invertido, Donatti, U horizontal, sutura contínua e intradérmica, compuseram seis estações de avaliação. Após a prática na bancada, os discentes vivenciaram situação real em ambiente ambulatorial. Os discentes foram avaliados com conceitos bom e regular, inclusive na prática ambulatorial, mostra que o método de sutura em consistências diferentes favorece a aprendizagem. A estratégia utilizando material com diversas texturas mostrou-se efetivo para desenvolver habilidades e aquisição de competências para prática de sutura de pele. A oficina despertou interesse para especialidade cirúrgica.
\end{abstract}

Palavras-chave: Educação Médica; Materiais de Ensino; Modelos Educacionais; Cirurgia Técnicas de Sutura; Procedimentos Cirúrgicos Ambulatoriais.

\begin{abstract}
The objective shows the potentialities of a bench model using organic and synthetic materials, designed to development and skill acquisition in skin suturing. Method: Case study with students of the medical school. A workshop was held to practice skin sutures, providing basic suture techniques with simulated training in three tissue consistencies. Data were collected from the application of questionnaires and observation. After practicing in this bench model, the students had the opportunity to experience real situation in an outpatient setting. Results: On the workbench the sutures were made in the following materials EVA, eggplant and ox tongue, using simple point, simple inverted, Donatti, U horizontal, continuous and intradermal suture. The results show that practice in different consistencies favors learning. Conclusion: The teaching strategy proved effective for the development of skills and acquisition of skills necessary for the practice of skin suture. The workshop aroused interest for the surgical specialty.
\end{abstract}

Keywords: Medical Education; Surgery; Suturing Techniques; Outpatient Surgical; Educational Model.

\footnotetext{
${ }^{1}$ Mestra em Ensino na Saúde pela Universidade Federal de Alagoas (UFAL). Preceptora Faculdade de Medicina da Universidade Federal de Alagoas (UFAL), Maceió, Alagoas, Brasil. E-mail: emmanuella.oliveira@gmail.com

${ }^{2}$ Doutora em Educação pela Pontificia Universidade Católica de São Paulo (PUC/SP). Professora do Programa de Pós-Graduação Mestrado Profissional Ensino na Saúde da Universidade Federal de Alagonas (UFAL). Maceió, Alagoas, Brasil. E-mail: lenildaaustrilino@gmail.com
} 


\section{Introdução}

As Diretrizes Curriculares Nacional (DCNs) estabelecem que a formação médica deva prover aos discentes, habilidades, competências e conhecimentos inerentes ao médico generalista (BRASIL, 2014). Define atenção à saúde, tomada de decisões, comunicação, administração e gerenciamento e, educação permanente como competências gerais que devem ser adquiridas durante os cursos de graduação. Requer destreza na integração entre os conteúdos das diversas especialidades médicas. Orienta para a utilização de metodologias que privilegiem a participação ativa do aluno na construção do conhecimento e a integração entre os conteúdos, além de estimular a interação entre o ensino, a pesquisa e a extensão/assistência; relaciona os conhecimentos, competências e habilidades específicas que os graduandos devem ter e menciona que a avaliação deverá basear-se nas competências, habilidades e conteúdos curriculares.

Entre os conhecimentos, habilidades e competências específicas, as DCNs estabelecem que os discentes realizem procedimentos clínicos e cirúrgicos, indispensáveis para o atendimento ambulatorial e para o atendimento das urgências e emergências. Para tal, indica que noções básicas - teóricas e práticas - de técnica cirúrgica são indispensáveis para todos os discentes do curso de medicina, mesmo para aqueles que não pretendam dedicar-se às especialidades cirúrgicas, uma vez que a formação é voltada para o médico generalista. Assim, todos devem ter o domínio técnico de procedimentos cirúrgicos utilizados em situações de urgência/emergência.

Em consonância com as DCNs o Projeto Pedagógico do Curso de Medicina da Universidade Federal de Alagoas - PPC/UFAL (UFAL, 2010) em sua matriz curricular estabelece entre os objetivos de aprendizagem para o ciclo teórico - prático: iniciar a prática do atendimento global e acompanhamento do politraumatizado e das emergências clínico cirúrgicas na criança, adultos e idosos; realizar procedimentos de suporte básicos e avançados de vida e pequenas cirurgias ambulatoriais; e, desenvolver habilidades psicomotoras para realizar intervenções clínico cirúrgicas de urgência e emergência.

Porém, o tempo dedicado à conciliação teoria prática, as dificuldades de recursos humanos e materiais requer o desenvolvimento de estratégias de ensino que potencializem o adequado domínio das técnicas e o alcance dos objetivos propostos. Os conteúdos da disciplina prática cirúrgica estão entre aqueles que necessitam de treinamentos de bancada, em simulações que se aproximem ao máximo de situações reais, promovendo o desenvolvimento de habilidades e competências que favoreçam a 
aquisição de destreza para a realização de intervenções clínico cirúrgica de urgência e emergência (OLIVEIRA; AUSTRILINO, 2018).

Tradicionalmente, o ensino de cirurgia se iniciava com a prática da observação, animais ou cadáveres eram utilizados para treinar as técnicas, de modo a aproximar ao máximo o modelo da realidade. Com as discussões sobre bioética foram introduzidos modelos alternativos, eticamente aceitos. Há uma variedade de modelos propostos para o desenvolvimento de habilidades em práticas cirúrgicas que estão sintonizados com a complexidade do processo ensino aprendizagem, com o desenvolvimento tecnológico e com as questões éticas contemporâneas. São modelos que utilizam como recurso educativo materiais sintéticos, materiais orgânicos, softwares, vídeos ilustrativos, programas interativos, entre outros.

O modelo de bancada mais utilizado nos programas de residência médica em dermatologia nos EUA é a pata de porco por simular a pele humana (PURIM, 2010). Com propósito semelhante, outros modelos utilizam animais post-mortem, principalmente pele de galinha (DENADAI; SOUTO, 2012) e língua bovina, (FRANCO et al., 2008). Há também a alternativa de usar materiais orgânicos como frutas e legumes (DENADAI; SOUTO, 2012). Amber van Drever et al., (2018) propuseram utilizar uma cultura simbiótica de bactérias e leveduras, designada SCOBY (DREVEN, 2018) como alternativa viável para treinamento de sutura e excisão, devido a sua semelhança com a pele, tanto em relação a sutura como ao corte. Segundo eles "Com exceção do odor, os entrevistados classificaram o SCOBY superior ao EVA e equivalente ao porco".

A participação ativa do discente na utilização de modelos de bancada é importante para o desenvolvimento de habilidades e para o domínio da prática antes de executá-la em ambiente real. Independente do modelo utilizado há um conjunto de aspectos a serem avaliados na perspectiva de identificar o grau de conhecimento adquirido e o desempenho nas habilidades requeridas durante a realização dos treinamentos em bancada.

De acordo com Hauser e Bowen (2009), para os iniciantes, instruções explicitas e fornecimento de feedback sobre o desempenho, são estratégias que facilitam a aprendizagem de habilidades motoras. Repetição e prática contínua melhoram o desempenho, bem como a avaliação pautada em listas de classificação com critérios específicos relacionados a cada etapa do treinamento, pode ser uma ferramenta valiosa para avaliado e avaliador. Além da avaliação segundo critérios preestabelecidos, os estudantes devem fazer auto avaliação e receber, após cada fase do treinamento, 
feedebacks individuais (PURIM, 2010) visando identificar qualidades, erros e deficiências nos movimentos específicos executados para cada técnica.

Por meio do feedback construtivo, os alunos podem aperfeiçoar suas habilidades, praticar repetidamente e consequentemente desenvolver habilidades ao longo do tempo (DENANDAI; SOUTO, 2012). O quadro a seguir, mostra a Global Rating Scale (GRS), uma escala validada internacionalmente na qual é possível identificar aspectos genéricos do desempenho dos participantes, em oito das principais áreas da prática cirúrgica.

Quadro 1: Global Rating Scale - para avaliação da aquisição de habilidades cirúrgicas

\begin{tabular}{|c|c|c|c|c|c|}
\hline Respeiro para o & 1 & 2 & 3 & 4 & 5 \\
\hline & $\begin{array}{l}\text { Usou frequentememente } \\
\text { força desnecessária no } \\
\text { tecido ou gerou danos } \\
\text { pelo uso inadequado dos } \\
\text { instrumentos } \\
\end{array}$ & & $\begin{array}{c}\text { Manuseio cuidadoso } \\
\text { do tecido, mas } \\
\text { ocasionalmente gerou } \\
\text { danos }\end{array}$ & & $\begin{array}{l}\text { Tecidos manipilados } \\
\text { adequadamente, com } \\
\text { dados mínimos }\end{array}$ \\
\hline \multirow[t]{2}{*}{$\begin{array}{c}\text { Tempo no } \\
\text { movimento }\end{array}$} & 1 & 2 & 3 & 4 & 5 \\
\hline & $\begin{array}{l}\text { Tempo insatisfatório / } \\
\text { Muitos movimentos } \\
\text { desnecessários }\end{array}$ & & $\begin{array}{c}\text { Tempo e movimento } \\
\text { eficientes, mas alguns } \\
\text { movimentos } \\
\text { desnecessários }\end{array}$ & & $\begin{array}{l}\text { Clara economia de } \\
\text { movimento e } \\
\text { máxima eficiência }\end{array}$ \\
\hline \multirow{2}{*}{$\begin{array}{c}\text { Manipulação de } \\
\text { instrumento }\end{array}$} & 1 & 2 & 3 & 4 & 5 \\
\hline & $\begin{array}{c}\text { Fez repetidamente } \\
\text { movimentos inábeis ou } \\
\text { hesitantes, através da } \\
\text { utilização inadequada dos } \\
\text { instrumentos }\end{array}$ & & $\begin{array}{c}\text { Utilizou de forma } \\
\text { competente os } \\
\text { instrumentos, mas, as } \\
\text { vezes pareceu } \\
\text { inflexível (rígido) ou } \\
\text { desajeitado (inábil) }\end{array}$ & & $\begin{array}{c}\text { Manipulação } \\
\text { adequada dos } \\
\text { instrumentos, sem } \\
\text { dificuldades }\end{array}$ \\
\hline \multirow{2}{*}{$\begin{array}{l}\text { Técnica de } \\
\text { sutura* }\end{array}$} & 1 & 2 & 3 & 4 & 5 \\
\hline & $\begin{array}{c}\text { Desajeitado e inseguro, } \\
\text { amarrando os nós } \\
\text { inadequadamente e } \\
\text { incapacidade para manter } \\
\text { a tensão }\end{array}$ & & $\begin{array}{l}\text { Cuidadoso e lento, } \\
\text { com maioria dos nós } \\
\text { colocados } \\
\text { adequadamente com } \\
\text { tensão adequada }\end{array}$ & & $\begin{array}{l}\text { Excelente controle } \\
\text { da sutura com } \\
\text { colocação adequada } \\
\text { dos nós e correta } \\
\text { tensão }\end{array}$ \\
\hline \multirow{2}{*}{$\begin{array}{l}\text { Técnica de } \\
\text { biopsia } \\
\text { excisional } \\
\text { elíptica** }\end{array}$} & 1 & 2 & 3 & 4 & 5 \\
\hline & $\begin{array}{c}\text { Falta conhecimento sobre } \\
\text { os parâmetros }(<2 \mathrm{~mm} \text { ou } \\
>10 \mathrm{~mm} \text { de margens }), \\
\text { ângulos muito diferente do } \\
\text { que } 30^{\circ} \text {, relação } \\
\text { comprimento-largura } \\
\text { muito diferente do que } 3 \text { - } \\
4: 1\end{array}$ & & $\begin{array}{c}\text { Margens adequadas } \\
\text { (2-10mm), ângulos } \\
\text { nas extremidades da } \\
\text { elipse ligeiramente } \\
\text { diferentes que } 30^{\circ} \text {, } \\
\text { relação comprimento- } \\
\text { largura diferente do } \\
\text { que 3-4:1 }\end{array}$ & & $\begin{array}{c}\text { Margens adequadas } \\
(2-10 \mathrm{~mm}) \text {, ângulos } \\
\text { nas extremidades da } \\
\text { elipse de } 30^{\circ} \text {, relação } \\
\text { comprimento-largura } \\
\text { de } 3-4: 1\end{array}$ \\
\hline \multirow[t]{2}{*}{$\begin{array}{l}\text { Fluxo da } \\
\text { operação }\end{array}$} & 1 & 2 & 3 & 4 & 5 \\
\hline & $\begin{array}{l}\text { Frequentemente hesitou } \\
\text { na execução do } \\
\text { procedimento e parecia }\end{array}$ & & $\begin{array}{l}\text { Demonstrou algum } \\
\text { planejamento para a } \\
\text { execução do }\end{array}$ & & $\begin{array}{l}\text { A operação foi } \\
\text { executada com } \\
\text { eficiência, com }\end{array}$ \\
\hline
\end{tabular}




\begin{tabular}{|c|c|c|c|c|c|}
\hline & $\begin{array}{c}\text { incerto (ou inseguro) } \\
\text { quanto ao próximo passo }\end{array}$ & & $\begin{array}{l}\text { procedimento, com } \\
\text { progressão razoável } \\
\text { dos passos } \\
\end{array}$ & & $\begin{array}{c}\text { progressão adequada } \\
\text { de um movimento } \\
\text { para outro }\end{array}$ \\
\hline \multirow{2}{*}{$\begin{array}{l}\text { Conhecimento } \\
\text { do } \\
\text { procedimento } \\
\text { específico } \\
\end{array}$} & 1 & 2 & 3 & 4 & 5 \\
\hline & Conhecimento deficiente & & $\begin{array}{c}\text { Tem noção de todas as } \\
\text { etapas importantes da } \\
\text { operação }\end{array}$ & & $\begin{array}{c}\text { Demonstrou } \\
\text { familiaridade com } \\
\text { todas as etapas da } \\
\text { operação }\end{array}$ \\
\hline \multirow[t]{2}{*}{$\begin{array}{l}\text { Qualidade do } \\
\text { produto final }\end{array}$} & 1 & 2 & 3 & 4 & 5 \\
\hline & Muito pobre & & Competente & & Excepcional \\
\hline \multirow[t]{2}{*}{$\begin{array}{c}\text { Desempenho } \\
\text { global }\end{array}$} & 1 & 2 & 3 & 4 & 5 \\
\hline & Muito pobre & & Competente & & Excepcional \\
\hline $\begin{array}{l}\text { Máximo de } \\
\text { pontos } \\
\text { Marque a nota } \\
\text { final }\end{array}$ & & & & & $\begin{array}{l}(40) \\
(\quad)\end{array}$ \\
\hline
\end{tabular}

Fonte: (DENADAI et al., 2014)

O quadro 1 organiza os critérios de avaliação da aquisição de habilidade cirúrgicas, atribuindo escores para a sequência de procedimentos, sem necessidade de desenvolver listas específicas para cada um deles (KHAN et al., 2007). Os principais aspectos avaliados são: respeito ao tecido; tempo de movimento; manipulação do instrumento; técnica de sutura; técnica de biopsia excecional elíptica; fluxo de operação; conhecimento do procedimento específico e desempenho global.

Visando proporcionar uma aprendizagem significativa (AUSUBEL, 1978) em técnicas cirúrgicas, esta pesquisa foi desenvolvida com o objetivo de identificar as potencialidades de um método de ensino, utilizando materiais orgânicos e sintéticos de diferentes consistências. Método delineado na intenção de propiciar o desenvolvimento de habilidades e aquisição de competências em sutura de pele. O presente trabalho visa mostrar as potencialidades do modelo de bancada proposto, como estratégia de ensino inovadora, na graduação em medicina.

\section{Metodologia}

Pesquisa na modalidade estudo de caso (LÜDKE, 2016; YIN, 2010; DEUS; CUNHA; MACIEL, 2010) visando compreender a ação educativa, no que concerne a habilitação de graduandos do curso de medicina para a realização de procedimento cirúrgico - sutura de pele, por ser um método abrangente, contextualizado e de "grande 
utilidade em pesquisas exploratórias e comparadas" (VENTURA, 2007, p. 386). O caso a ser pesquisado surge da necessidade de verificar como treinar estudantes visando a aquisição de habilidades e competências, para realizarem sutura de pele em ambiente ambulatorial.

\begin{abstract}
Descrever e caracterizar estudos de caso não é uma tarefa fácil, pois eles são usados de modos diferentes, com abordagens quantitativas e qualitativas, não só na prática educacional, mas também como modalidade de pesquisa, com aplicação em muitos campos do conhecimento, principalmente na Medicina, Psicologia e em outras áreas da saúde [...] (VENTURA, 2007, p. 383).
\end{abstract}

A estratégia de ensino inovadora intitulada "Oficina de sutura de pele" utilizou um modelo de bancada, composto com material sintético e orgânico de natureza vegetal e animal, seguido de uma prática supervisionada em ambulatório. A inovação proposta constou da utilização de materiais com consistências diferentes, da aplicação de diversas técnicas de sutura, e da oportunidade de os discentes aplicarem o aprendido, realizando sutura em ambulatório sob a supervisão da pesquisadora.

A atividade foi realizada no período de 15 de setembro a 7 de novembro de 2016, com encontros semanais, totalizando 20 horas de treinamento, mais quatro horas de prática ambulatorial. Participaram doze discentes da graduação em medicina, sendo seis do $4^{\circ}$ período, e seis do $10^{\circ}$ período. Os discentes trabalharam em duplas, um do $4^{\circ}$ e outro do $10^{\circ}$ período, na proporção de um tutor para cada discente, (ALBUQUERQUE et al., 2012) acompanhando diretamente a execução de cada técnica, observando, estimulando, esclarecendo dúvidas e auxiliando na condução do processo, todos sob a supervisão da pesquisadora.

Inicialmente, os discentes foram orientados quanto aos aspectos operacionais da oficina e preenchimento de questionários, recebendo kits cirúrgicos individualizados contendo: tesoura, pinça anatômica, pinça dente de rato, porta agulhas, cabo e lâminas de bisturi, fios agulhados e luvas de procedimento.

O roteiro dos exercícios práticos incluía seis estações de sutura de pele, em cada uma delas havia três texturas diferentes: sintético - EVA e orgânico - berinjela e língua de boi. Cada exercício foi precedido de explicação detalhada, bem como demonstração em multimídia. As estações correspondiam a cada um dos tipos de suturas propostas, ou seja: estação 1 - ponto simples; estação 2 - ponto simples invertido; estação 3 - ponto em U horizontal; estação 4 - ponto em U vertical (Donatti); estação 5 - sutura contínua e estação 6 - sutura intradérmica. Em cada estação os itens avaliados foram concernentes 
a: respeito ao tecido; tempo de movimento; manipulação do instrumento e técnica de sutura.

As suturas realizadas em cada estação foram avaliadas por observação dos discentes/tutores e pela docente/pesquisadora, sendo atribuído um conceito bom, regular ou insuficiente de acordo com os critérios de avaliação de desempenho, elaborados a partir dos parâmetros validados, pela escala de observação GRS, para cada uma das texturas utilizadas.

Os critérios observados foram: colocação do fio no porta agulhas, profundidade do ponto, distância do fio a margem da ferida, distância entre os pontos, empunhadura do porta agulhas, uso da pinça, realização do nó, tensão do nó da sutura, eversão dos bordos, coaptação dos bordos, destreza na realização da sutura, corte do fio e tempo de realização da sutura. As pontuações atribuídas, somadas, resultaram nos escores obtidos. O tempo de realização das suturas também foi registrado nas fichas individuais de avaliação.

O quadro a seguir, mostra a planilha utilizada para acompanhamento do desempenho do discente durante o período de realização do treinamento. Ao final da oficina eles foram para o centro cirúrgico de pequenas cirurgias e supervisionados pela docente/pesquisadora realizaram suturas em pacientes.

Quadro 2: Planilha de acompanhamento do desempenho discente

\begin{tabular}{|l|l|l|l|l|l|l|l|l|l|l|l|l|l|l|l|l|l|l|}
\hline Estação & \multicolumn{3}{|c|}{1} & \multicolumn{3}{|c|}{2} & \multicolumn{3}{c|}{3} & \multicolumn{3}{c|}{4} & \multicolumn{3}{c|}{5} & \multicolumn{3}{c|}{6} \\
\hline Textura & 1 & 2 & 3 & 1 & 2 & 3 & 1 & 2 & 3 & 1 & 2 & 3 & 1 & 2 & 3 & 1 & 2 & 3 \\
\hline Conceito & & & & & & & & & & & & & & & & & & \\
\hline Escore & & & & & & & & & & & & & & & & & & \\
\hline Tempo & & & & & & & & & & & & & & & & & & \\
\hline
\end{tabular}

Fonte: Elaboração da autora

Os questionários aplicados pré e pós-treinamento foram elaborados visando obter informações sobre as dimensões a serem avaliadas (MARCONDES et al., 2014). Compostos por perguntas abertas e fechadas, as quais buscaram identificar as expectativas dos estudantes com a oficina, o interesse pela área de cirurgia e, se possuíam habilidades em técnicas cirúrgicas, respondendo sim ou não a questões relacionadas aos conhecimentos prévios sobre a temática. A validação dos questionários se deu ao comparar o que foi respondido pelos participantes com o que foi observado em campo (MINAYO; COSTA, 2019). 
Ao final de cada encontro os discentes faziam autoavaliação identificando aspectos que os auxiliassem para um melhor desempenho. Os resultados das observações foram cotejados com as respostas aos questionários, aplicados pré e pós-treinamento e, com os resultados das autoavaliações, compondo assim um banco de dados das respostas dos sujeitos da pesquisa, permitindo comparar os resultados, assegurando maior de confiabilidade nas análises.

As respostas aos questionários foram sistematizadas, quadro 3, e analisadas individualmente, para cada sujeito da pesquisa - síntese horizontal -, e no coletivo, por questão - síntese vertical. Em seguida, foram destacadas ideias chaves na perspectiva de subsidiar a análise temática.

Quadro 3: Planilha demonstrativa da síntese das análises das repostas aos questionarios

\begin{tabular}{|c|c|c|c|c|}
\hline Sujeitos da pesquisa & Questão 1 & Questão 2 & Questão X & \\
\hline D1 & Resposta 1 de D1 & Resposta 2 de D1 & Resposta X de D1 & Síntese horizontal1 \\
\hline D2 & Resposta 1 de D2 & Resposta 2 de D2 & Resposta X de D2 & Síntese horizontal 2 \\
\hline Dn & Resposta 1 de Dn & Resposta 2 de Dn & Resposta X de Dn & Síntese horizontal n \\
\hline & Síntese vertical 1 & Síntese vertical 2 & Síntese vertical X & \\
\hline
\end{tabular}

Fonte: Elaboração autora

Os dados provenientes das diferentes fontes foram entrecruzados e analisados na perspectiva da análise temática, visando identificar evidências de aquisição de habilidades para sutura de pele, de acordo com os critérios estabelecidos na GRS. Os participantes foram identificados pela letra $\mathrm{D}$ seguido de número. A pesquisa foi submetida ao comitê de ética parecer $\mathrm{n}^{\circ}$ 1718134. A oficina também considerou a legislação sanitária para descarte de material orgânico e perfurocortante.

\section{Resultados e discussão}

Em questionário aplicado pré-treinamento os discentes responderam que participaram da Oficina com a expectativa de "aprender o máximo, visto que esse é um momento raro da rotina acadêmica antes do internato" (D5). "Aprender as técnicas corretas ... ter experiência prévia antes de chegar ao paciente” (D2). Quatro dos participantes informaram ter assistido a algum tipo de cirurgia, três possuíam conhecimento sobre suturas com ponto simples, e um, além do ponto simples, tinha conhecimento sobre sutura contínua. Exceto um discente, os demais tinham interesse na especialidade cirúrgica. 
Durante o treinamento os discentes apontaram dificuldades com o manuseio do material: "No início foi difícil o manuseio dos instrumentos, principalmente o porta agulha, com o passar da prática a habilidade foi melhorando consegui ver melhoras (sic) inclusive na qualidade dos pontos" (D6). Entre as texturas disponíveis, a do EVA e a da berinjela ofereceram maior dificuldade para a execução da sutura: "no uso da berinjela tive alguma dificuldade em manter a delicadeza, o fio estourou algumas vezes” (D2) “... tive mais dificuldade com o emborrachado, mais facilidade com a língua de boi, com a berinjela tive alguma dificuldade...” (D5). "A berinjela para o ponto simples atende bem as necessidades didáticas" (D3).

Os materiais utilizados na oficina para o modelo de bancada possuíam texturas diferentes visando desenvolver habilidades de coordenação motora e regular o uso da força para as diferentes resistências dos tecidos, uma vez que tecidos friáveis vão requerer uma coordenação motora diferenciada. Para Denadai e Souto (2012), a fidelidade do modelo não é importante, fundamental é a transferência da habilidade do ambiente de laboratório para a prática clínica. A transferência das habilidades desenvolvidas durante a repetição dos seis tipos de ponto/sutura em texturas diferentes favoreceu a evolução nos estágios de competências, haja vista o desempenho durante a prática ambulatorial.

O feedback imediato após cada estação facilitou a progressão e aperfeiçoamento das habilidades: “... quando tive dificuldade a minha dupla interno (sic) sempre me ajudou a melhorar" (D4). A apresentação imediata do feedback, estimula o discente mesmo quando ele não acerta, pois proporciona a chance de revisar, refazer e de aprender com o erro. $\mathrm{O}$ fato de ter um tutor para cada estudante também contribuiu para agilizar a avaliação, devido a rapidez no feedback. Associado ao retorno da avaliação, a interlocução para dirimir as dúvidas, durante todo o processo, contribuiu para aperfeiçoar a técnica.

Das observações feitas, na avaliação dos tutores, os alunos obtiveram conceito entre regular e bom, havendo uma melhora progressiva no decorrer da oficina, com diminuição do tempo para a execução das tarefas propostas, bem como aprimoramento da técnica, aspecto também relatado por um dos discentes.

Consegui aprimorar a técnica ... como a maneira de segurar o porta agulhas, aproximar as bordas a partir do meio para diminuir a tensão, consegui melhorar o nó com a prática e deixar o ponto simétrico com o mesmo espaçamento na tensão correta (D3).

Os discentes participantes afirmaram ter evoluído na aprendizagem: "pude treinar várias vezes em diferentes formas de tecidos o que me proporcionou aprimorar a técnica" 
(D5); "pude aprender e aprimorar com a repetição" (D6); "Foi muito bom consegui fazer a técnica e treinar, com a repetição pude aprender" (D1). A repetição é outro fator importante na aquisição de habilidades em técnicas cirúrgicas. Foram seis estações em três texturas diferentes totalizando 18 momentos de contato com a técnica e com a oportunidade de desenvolvimento de habilidades e de evolução dos estágios de competência.

Fitts e Posner (1967) e Chambers (1998) defendem que a aprendizagem motora acontece em estágios ou etapas, onde o aprendiz passa por diferentes situações desde o primeiro contato com o novo movimento até dominá-lo completamente. Para Chambers (1998) em sua teoria das habilidades motoras, os estágios de desenvolvimento motor são: novato, iniciante, competentes, proficiente e perito. Considerando as observações feitas os estudantes atingiram o estágio de competentes (HAUSER; BOWEN, 2009), ou seja, prontos para começar a prática de maneira independente, com supervisão.

Embora tenham praticado várias vezes em situação de laboratório, as suturas realizadas em ambiente real, em ambulatório com paciente, a avaliação de desempenho feito pela docente/pesquisadora mostrou os aspectos em que os discentes apresentaram alguma dificuldade. No ambulatório todos os alunos receberam conceito regular, pelo uso da pinça, o que se justifica por exigir o uso da mão não dominante, o que requer mais treinamento. No item referente a coaptação dos bordos, 4 dos 6 discentes receberam o conceito regular. O desempenho regular pode ser justificado devido ao fato de ser o primeiro contato em situação real, em um ambiente onde eles não estão habituados, e também devido a presença da docente/pesquisadora e do paciente.

Comparando as resposta dos questionários pós-treinamento com as do prétreinamento, os discentes avaliaram que a oficina despertou o interesse pela especialidade, destacando o discente (D6), que inicialmente respondeu; "não tenho interesse em seguir a especialidade cirúrgica, desejo melhorar a formação inicial" e no pós-treinamento afirma: "o curso foi além do que eu esperava. Despertou o interesse, mostrando ser mais prazeroso do que imaginava" (D6). Stain et al, 2013, dizem que a exposição de estudantes ao âmbito cirúrgico influência de maneira positiva a escolha pela carreira. Os resultados obtidos com o desempenho dos discentes no centro cirúrgico mostram que a estratégia de ensino favoreceu a aquisição e transferência de habilidades para a prática clínica.

Além disso, os discentes avaliaram positivamente o treinamento realizado, segundo eles: "o treinamento em diferentes texturas contribui para perceber os diferentes 
aspectos relacionados a sutura, simetria, força e outros. Adorei" (D4). "Muito importante para aprender pontos mais estéticos, e mais sofisticados, aumentar a prática com os nós e a técnica correta da mão" (D1). O ensino aprendizagem das habilidades cirúrgicas básicas é de interesse especial, pois estas competências são o alicerce sobre o qual as demais aptidões serão construídas.

Para auxiliar na aprendizagem, favorecendo a autonomia e respeitando as diferentes formas com que os discentes constroem seus conhecimentos, um e-book ${ }^{3}$ foi elaborado pela docente/pesquisadora, como produto resultante das reflexões feitas durante a oficina. Composto por vídeos, textos explicativos sobre as técnicas de sutura de pele praticadas, nas diversas consistências utilizadas.

\section{Conclusão}

Os resultados desta pesquisa mostram que o modelo de bancada proposto para sutura de pele é eficaz e contribui para a aprendizagem em práticas cirúrgicas. A estratégia de ensino é inovadora devido a conformação da bancada em estações. Utilizar materiais de consistências diferentes, favoreceu aos discentes treinar o manuseio dos instrumentos e, identificar que cada textura requer coordenação motora e regulação diferenciada do uso da força, habilidades requeridas e imprescindíveis para a realização de suturas.

A análise das respostas dos estudantes aos questionários pré e pós treinamento, as observações feitas durante a oficina e, as avaliações dos próprios estudantes sobre as suturas por eles realizadas permitiram a reflexão qualitativa de que a Oficina de Sutura de Pele foi um momento de aprendizagem, para os discentes participantes, que transpuseram o treinamento de bancada e realizaram sutura de pele em ambulatório com paciente. Para a docente/pesquisadora o processo de pesquisa favoreceu a sistematização de um e-book, como produto das reflexões advindas das potencialidades do modelo de bancada utilizado. A oficina também demonstrou ser motivadora para a escolha da especialidade cirúrgica.

Este estudo teve como limitação o uso exclusivo da técnica de sutura de pele, entretanto, os resultados poderão subsidiar novas investigações e proposições de práticas pedagógicas contemplando o desenvolvimento e aquisição de habilidades para a realização de outras técnicas cirúrgicas, por exemplo, incisão e enxertos. Mudanças nas

\footnotetext{
${ }^{3} \mathrm{O}$ ebook está disponível no site http://www.ufal.edu.br/unidadeacademica/famed, sendo de livre acesso a qualquer pessoa interessada no assunto.
} 
formas de avaliação e no processo ensino aprendizagem, tanto na formação inicial como continuada, de médicos e enfermeiros que necessitam adquirir essas habilidades, poderão ser introduzidas a partir das conclusões resultantes desta pesquisa.

\section{Referências}

ALBUQUERQUE, G. et al. M. Monitoria de técnica operatória e cirurgia experimental e sua relevância na formação médica. Revista Brasileira de Educação Médica, Rio de Janeiro, v. 36, n. 4, p. 564-569, 2012.

AUSUBEL, D. P. Educational Psychology: a Cognitive View. 1. ed. Nova York: Holt, Rinehart and Winston, 1968.

BRASIL. Ministério da Educação. Diretrizes Curriculares Nacional para Área da Saúde. Brasília, 2014

CHAMBERS, D. Competency-based dental education in context. European Journal of Dental Education, EUA, v. 2, n. 1, p. 8-13, jan. 1998.

DENADAI, R.; SOUTO, L. Organic bench model to complement the teaching and learning on basic surgical skills. Acta Cirúrgica Brasileira, São Paulo, v. 27, n. 1, p. 88-94, jan. 2012.

DENADAI, R. et al. Low fidelity bench models for basic surgical skills training during undergraduate medical education. Rev. Col. Bras. Cir. Dr. Mario Gatti County Hospital Postgraduation Program in General Surgery, São Paulo. v. 41, n. 3, p. 137-145, may. 2014.

DEUS, A.; CUNHA, D.; MACIEL, E. Estudo de caso na pesquisa qualitativa em educação: uma metodologia. In: ENCONTRO DE EDUCAÇÃO, 6., 2010, São Paulo. Atas... São Paulo: UNESP, 2010. p. 5129.

DREVEN, V. A. et al. Will SCOBY do? An alternative for teaching basic surgical skills of suturing and skin excision, Journal of Surgical Simulation, Washington, v. 5, n. 99, p. 99104, jan. 2018. Disponível em: https://doi.org/10.1102/2051-7726.2018.0012. Acesso em: 04 fev. 2018.

FITTS, P. M.; POSNER, M. I. The Cognitive Stage of Learning. In: EDWARDS, W. H. Motor Learning and control: from Theory to Practice. Taiwan: Cenage Learning, 2010.

FRANCO, D. et al. Uso de língua bovina na prática de técnicas de sutura. Rev. Col. Bras. Cir. Rio de Janeiro, v. 35, n. 6, p. 442-444, nov./dec. 2008.

HAUSER, A.; BOWEN, D. Primer on preclinical instruction and evaluation. Journal of dental education, Washington, v. 73, n. 3, p. 390-398, may. 2009.

KHAN, M. et al. Assessing Surgical Skill Using Bench Station Models. Plastic and Reconstructive Surgery, [S.I.], v. 120, n. 3, p. 793-800, out. 2007.

LÜDKE, M. Educação e pesquisa qualitativa no Brasil. In: SOUZA, D. N.; COSTA, A. P.; SOUZA, F. N. Investigação Qualitativa: inovação, dilemas e desafios. 1. ed. Porto: Ludomedia, 2016. p. 77- 104. 
MARCONDES, C., PESSOA, S., \& et al. Sistematização do treinamento teórico e prático de técnicas em suturas para acadêmicos de medicina da disciplina de cirurgia plástica da Universidade Federal do Ceará - UFC. Revista Brasileira de Cirurgia Plástica, São Paulo, v. 29, n. 2, p. 289-293, 2014.

MINAYO, M. C. de S.; COSTA, A. P. Técnicas que Fazem Uso da Palavra, do Olhar e da Empatia: Pesquisa Qualitativa em Ação. 1. ed. Portugal: Ludomedia, 2019.

OLIVEIRA, E. A.; AUSTRILINO, L. Estratégia de ensino para a prática de suturas de pele. In: CONGRESSO IBERO-AMERICANO EM INVESTIGAÇÃO QUALITATIVA, 7., 2018, Fortaleza. Atas... Portugal: Ludomedia, 2018. p. 570-577.

PURIM, K. S. M. Oficina de cirurgia cutânea. Rev. Col. Bras. Cir, [S.I.], v. 37, n. 4, p. $303-$ 305, 2010.

UNIVERSIDADE FEDERAL DE ALAGOAS (UFAL), Projeto Pedagógico do Curso de Medicina. Alagoas: EDUFAL, 2010

VENTURA, M. M. O Estudo de Caso como Modalidade de Pesquisa. Rev. SOCERJ., Rio de Janeiro, v. 20, n. 5, p. 383-386, set./out. 2007.

YIN, R. Estudo de Caso: Planejamento e Métodos. 2. ed. Porto Alegre: Bookman, 2010.

Recebido em: 01 de março de 2019.

Aceito em: 26 de julho de 2019. 